\title{
DEGRADABILIDAD RUMINAL DEL FORRAJE DISPONIBLE EN LA PRADERA Y DEL APARENTEMENTE CONSUMIDO POR VACAS LECHERAS ${ }^{1}$
}

\author{
RUBÉN PULIDO² y JOHNDAVID LEAVER ${ }^{3}$
}

\begin{abstract}
RESUMEN - Se evaluó la dinámica degradativa de forrajes, en muestras de una pradera permanente (Lolium perenne L.), manejadas a dos alturas de pradera, alta y baja. Las muestras consistieron en forraje disponible a ras de suelo (FD) y en forraje aparentemente consumido por vacas lecheras en pastoreo continuo (FS). La degradabilidad ruminal se estudió usando la técnica de las bolsas de dacrón y los datos fueron ajustados a una ecuación exponencial. La fracción soluble ( $\mathrm{g} / \mathrm{kg} \mathrm{MS})$, de la materia seca (MS) $(299 \mathrm{v} / \mathrm{s} 351$, s.e.d. $=5,4)$, de la materia organica $(M O)(304 \mathrm{v} / \mathrm{s} 376$, s.e.d. $=3,3)$ y del nitrógeno $(250 \mathrm{v} / \mathrm{s} 301$, s.e.d. $=6,4)$, fueron significativamente mayores $(\mathrm{P}<0,05)$ en muestras de FS que en muestras de FD. La degradabilidad potencial ( $\mathrm{g} / \mathrm{kg} \mathrm{MS}$ ) de MS, MO y nitrógeno, fue significativamente mayor $(\mathrm{P}<0,05)$ en las muestras de FS, que en las muestras de FD. La degradabilidad efectiva fue mayor en las muestras de FS, que en las muestras de FD, tanto para la MS $(474 \mathrm{v} / \mathrm{s} 508$, s.e.d. $=13,0)$, MO $(490 \mathrm{v} / \mathrm{s} 529$, s.e.d.=11,6) y nitrógeno $(351 \mathrm{v} / \mathrm{s} 419$, s.e.d.=10,0). Respecto del factor altura, sólo se encontró un efecto significativo en la degradabilidad efectiva en el nitrógeno, en favor de la altura baja $(365 \mathrm{v} / \mathrm{s} 406$, s.e.d. $=10,0)$.
\end{abstract}

Términos para índice: muestras de forraje, pastura, bovino, degradación ruminal.

\section{RUMEN DEGRADATION CHARACTERISTICS OF THE HERBAGE MASS SAMPLES AND THE SIMULATED GRAZING SAMPLES FOR DAIRY COWS}

\begin{abstract}
An experiment was carried out in order to describe the ruminal degradation characteristics of forages, in herbage mass samples (FD) and in simulated grazing samples (FS), obtained at two sward heights (high and low) from a permanent pasture (Lolium perenne L.). The degradation kinetics of the samples was studied using in situ dacron bags. Data collected were fitted according to an exponential equation. The soluble fraction $(\mathrm{g} / \mathrm{kg} \mathrm{DM})$ of the dry matter $(\mathrm{DM})(299 \mathrm{vs.} 351$, s.e.d. $=5.4)$, of the organic matter $(\mathrm{OM})(304 \mathrm{vs.} 376$, s.e.d. $=3.3)$ and of the nitrogen $(250 \mathrm{vs.} 301$, s.e.d. $=6.4)$ were significantly higher $(\mathrm{P}<0.05)$ for simulated samples than for the herbage mass samples. The potential degradability ( $\mathrm{g} / \mathrm{kg} \mathrm{DM}$ ) of DM, OM and nitrogen was significantly higher on FS compared with the FD $(\mathrm{P}<0.05)$. The effective degradability for the FS samples was greater than for FD samples, either for DM (474 vs. 508, s.e.d.=13.0), OM (490 vs. 529 , s.e.d. $=11.6)$, or nitrogen $(351$ vs. 419 , s.e.d. $=10.0)$. A significant greater effect $(\mathrm{P}<0.05)$ of the low sward height on the effective degradability was found for the nitrogen only.
\end{abstract}

Index terms: forages sample, pasture, cattle, rumen degradation.

\section{INTRODUCCIÓN}

Los modelos predictivos que estiman la disponibilidad de nutrientes en los alimentos para los bovi-

\footnotetext{
${ }^{1}$ Aceptado para publicación en 23 de abril de 1999.

${ }^{2}$ Méd. Vet., Ph.D., Instituto de Zootecnia, Facultad de Ciencias Veterinarias, Universidad Austral de Chile, casilla 567, Valdivia, Chile. E-mail: rpulido@uach.cl

${ }^{3}$ Méd. Vet., Ph.D., Wye College, University of London, Wye, Ashford, Kent TN25 5AH, U.K.
}

nos, cada día involucran procedimientos más difíciles y complejos. Estos métodos han mostrado la necesidad de contar con características precisas de la cinética degradativa de las diferentes fracciones del alimento como parte indispensable del proceso de evaluación nutricional de los alimentos (Agricultural and Food Research Council, 1993).

Existe escasa información relacionada con la cinética degradativa ruminal del forraje pastoreado de diferentes calidades, tanto para el forraje total disponible que se le ofrece al animal en la pradera, así 
como para el que es realmente consumido por éste. Sin embargo, es posible esperar comportamientos distintos de estos forrajes en degradabilidad ruminal, debido a que el forraje realmente consumido en pastoreo contiene una mayor proporción de hojas verdes y menor proporción de materia muerta, con relación al forraje disponible (Black, 1990).

Mediciones con bolsas de dacrón han sido adoptadas como método estándar para determinar la degradabilidad de proteína del alimento (Agricultural and Food Research Council, 1993). El modelo generalmente usado es el propuesto por Orskov \& McDonald (1979).

El objeto de este estudio fue comparar las características de degradabilidad ruminal de forrajes en muestras provenientes del forraje disponible a ras de suelo en la pradera y en muestras de forraje aparentemente consumido por vacas lecheras en pastoreo, a dos alturas de pradera, de Lolium perenne L.

\section{MATERIAL Y MÉTODOS}

El experimento de degradabilidad in situ fue llevado a cabo en la Unidad Lechera de Wye College, Universidad de Londres, Kent, Inglaterra, desde el 12 al 30 de noviembre de 1995.

Se utilizaron tres novillos Hereford $\mathrm{x}$ Friesian provistos de una cánula ruminal. Los animales recibieron una ración de heno de pradera y un concentrado comercial (180 g PC/kg MS y $13.2 \mathrm{MJ} / \mathrm{kg}$ MS) en una proporción aproximada de $3: 1$. Las raciones fueron calculadas en orden de permitir un consumo diario de 1,0 - 1,5 veces el nivel de mantenimiento (Agricultural and Food Research Council, 1993). Los alimentos se ofrecieron en dos raciones iguales durante el día. Los animales disponían de libre acceso al agua.

Se tomaron muestras de forraje de una pradera permanente compuesta principalmente de gramíneas perennes (Lolium perenne), durante un experimento de pastoreo con vacas lecheras en la primavera. En este experimento (Pulido, 1997), la pradera fue manejada a dos alturas, bajo pastoreo continuo controlado (Set-stocking). Se estudió el forraje disponible a ras del suelo (FD) y el forraje aparentemente consumido por el animal en pastoreo (FS), ambos a dos alturas de pradera: altura pradera baja $(\mathrm{PB})$ y altura pradera alta (PA) (Cuadro 1). El forraje aparentemente consumido, se obtuvo cortando el forraje inmediatamente adyacente a aquel forraje consumido por el animal en pas- toreo. Este proceso de selección del forraje se realizó siguiendo al menos siete vacas por cada altura de pradera. Se utilizó una bolsa de incubación para cada animal (total de tres repeticiones) y por cada tiempo de incubación. Todas las muestras fueron secadas en un horno a $60^{\circ} \mathrm{C}$ por 48 horas, y luego se molieron en un molino de cuchillos con una criba de $1 \mathrm{~mm}$.

Para la incubación de las muestras, se usaron bolsas de dacrón con un tamaño estándar de $8 \mathrm{~cm}$ de largo por $5 \mathrm{~cm}$ de ancho, y con tamaño de poros de $43 \mu \mathrm{m}$. Todas las bolsas fueron lavadas, secadas, y pesadas antes de cada incubación. En cada bolsa previa identificación, se colocaron muestras de aproximadamente $5 \mathrm{~g}$ de cada forraje.

El proceso de incubación tuvo los siguientes tiempos: $0,8,12,24,48$ horas. Este se desarrolló de acuerdo al método de intercambio de inserción y remoción completa de bolsas (Paine et al., 1982).

Las bolsas se ataron a cadenas de acero inoxidable (un por animal) de $60 \mathrm{~cm}$ de largo, en la cual se dispusieron seis bolsas, permitiendo el movimiento de las bolsas en el interior del rumen. Luego de la incubación en los tiempos correspondientes, las bolsas fueron retiradas del rumen y sumergidas en agua fría por cinco minutos y después suavemente lavadas bajo agua corriente hasta que el agua de enjuague quedó aparentemente limpia. Luego del lavado, las bolsas fueron secadas en un horno a $60^{\circ} \mathrm{C}$ por un tiempo de 48 horas y pesadas. Los residuos de las bolsas fueron analizados separadamente o agrupadas por tiempo, si la cantidad remanente sobrante era inferior a $1 \mathrm{~g}$ para poder llevar a cabo el análisis de nitrógeno, cenizas y fibra detergente neutro $(\mathrm{FDN})$. Las determinaciones realizadas en todas las muestras de forraje fueron: materia seca (MS), proteína total (PT) y cenizas totales (CT) (Association of Official Analytical Chemists, 1980), fibra detergente neutro (FDN) y fibra detergente ácido (FDA) (Goering \& Van Soest, 1970), celulasa neutro detergente (NDC) (Jewell et al., 1986) y energía metabólica (EM) se estimó según Givens et al. (1990) y Inglaterra (1993).

Los resultados fueron usados para calcular la desaparición de la materia seca, materia orgánica (MO), fibra detergente neutro y degradación de nitrógeno.

La degradabilidad al tiempo 0 (fracción soluble) fue medida con tres muestras, en bolsas colocadas en agua tibia por 30 minutos. Después las bolsas fueron enjuagadas con agua fría como se señaló anteriormente. Luego fueron secadas y pesadas de manera de poder determinar la solubilidad.

Los datos de degradabilidad obtenidos fueron ajustados a la ecuación exponencial de Orskov \& McDonald (1979):

$\mathrm{p}=\mathrm{a}+\mathrm{b}(1-\exp (-\mathrm{ct}))$, donde: 
$\mathrm{p}=$ degradabilidad potencial de MS, MO, FDN y del N al tiempo t;

$\mathrm{a}=$ fracción soluble (a tiempo 0);

$\mathrm{b}=$ fracción insoluble, potencialmente degradable;

$\mathrm{c}=$ tasa fraccional de degradación de la fracción $\mathrm{b}$;

$\mathrm{t}=$ tiempo de incubación;

$\mathrm{a}+\mathrm{b}=$ degradación máxima potencial (asíntota).

Dado que el método in situ (sin corrección de tasa de pasaje) sobrestima los valores de degradabilidad (Agricultural and Food Research Council, 1993), se calculó la degradabilidad ruminal efectiva, al incorporar un valor de tasa de pasaje de $0,08 / \mathrm{h}$, equivalente a la tasa de pasaje del alimento por el rumen para vacas de alta producción. Esta se estimó de acuerdo a la ecuación:

$\mathrm{DE}=\mathrm{a}+\mathrm{bc} /(\mathrm{c}+\mathrm{k}) * \exp ((\mathrm{c}+\mathrm{k}) * \mathrm{t})($ McDonald, 1981) donde: $\mathrm{DE}=$ degradabilidad ruminal efectiva;

a, b y c $=$ valores determinados en la ecuación anterior;

$\mathrm{k}=$ tasa de fraccional de pasaje desde el rumen $(\mathrm{k}=0,08)$;

$\mathrm{t}=$ fase de retardo en la fermentación (lag time).

Con los datos se realizó un análisis de varianza en un diseño completamente al azar, con un arreglo factorial de $2 \times 2$, considerando tipo muestra de forraje obtenido (FD y FS) y altura de pradera (PA y PB), para evaluar si existieron diferencias en la cinética degradativa de las muestras de forraje. Estos análisis se realizaron utilizando el programa estadístico GenStat 5 Committee (1987).

\section{RESULTADOS Y DISCUSIÓN}

Es sabido que la composición del pasto depende de factores como especies, madurez, nivel de fertilización, suelo y tipo de condiciones climáticas, las que en parte pueden influir en las características de su degradabilidad en el rumen (Van Straalen \& Tamminga, 1990). Las muestras de forraje disponible a ras suelo FD mostraron concentraciones mayores de FDN y FDA y menores de proteína total que los del forraje aparentemente consumido (Cuadro 1). Es sabido que, los ovinos y bovinos a menudo seleccionan una dieta que pareciera tener poco parecido con la composición del total del alimento disponible (Dudzinki \& Arnold, 1973). En general, las ovejas tenderían a seleccionar en favor de una dieta que contiene una mayor proporción de hojas verdes y menor de tallos y materia muerta, con relación a la disponible en el forraje total (Black, 1990). Un patrón de comportamiento similar, pero menos pronunciado existe en el bovino (Forbes, 1986), el que aparecería mostrado en el proceso de selección del forraje aparentemente consumido por los bovinos fue adecuado.

Las características de la cinética de la degradación ruminal de la MS, MO, nitrógeno y FDN de las muestras de forraje se muestran en los Cuadros 2,3, 4 y 5 .

Las fracciones de degradación rápidas fueron en general bajas (a), lo que aparentemente se debió al procedimiento de lavado. Sin embargo, la fracción a y el potencial de degradación $a+b$ mostraron valores significativamente mayores $(\mathrm{P}<0,05)$ en el forraje aparentemente consumido, con relación al forraje disponible.

Las diferencias en degradabilidad de la MS (Cuadro 2) en el valor de lavado inicial a y el potencial de degradabilidad $(a+b)$ entre el FS y el FD pueden ser parcialmente explicadas por la diferencia de contenido de carbohidratos solubles o no estructurales. Von Keyserlingk et al. (1996) demostró que para un rango de forrajes, las diferencias encontradas para la fracción soluble de la MS, pueden ser atribuidas a diferencia en contenido de carbohidratos no estructurales. Junto a esto, la menor degradabilidad ruminal de la MS puede ser explicada por el aumento de fracciones indigeribles del forraje (Balde et al., 1993). Aunque la proporción hoja/tallo no fue medida en este experimento, es posible esperar un menor contenido de tallos en el forraje aparentemente consumido con relación al forraje disponible, y por lo tanto, un mayor contenido proporcional de carbohidratos no-estructurales. También, es esperable una menor cantidad de materia muerta en el FS, con respecto al FD. Si bien no hubo diferencias significativas $(\mathrm{P}>0,05)$ en la degradación efectiva de la MS, entre el FS y el FD, las muestras aparentemente consumidas tuvieron valores mayores.

Las características de degradación de la materia orgánica del forraje disponible y de las muestras simuladas son mostradas en el Cuadro 3. La degradación de la materia orgánica del FD y del FS mostró que la degradabilidad potencial $(a+b)$ fue significativamente mayor en las muestras aparentemente consumidas. Van Vuuren et al. (1991) informan que con Lolium perenne a comienzos de primavera, altos valores de a se encuentran asociados con bajos tenores de FDN y un aumento en la proporción 
de hoja/tallo. Los valores más bajos de a+b y de degradabilidad efectiva en el FD, pueden explicarse por sus mayores niveles de FDN y FDA, asociados probablemente a un aumento de la lignificación de la pared celular.

Las características de degradación del nitrógeno de los forrajes se muestran en el Cuadro 4. Los valores obtenidos de degradación coinciden con aquellos presentados por Agricultural and Food Research
Council (1993) y con el rango para a y b, de las muestras de pasto seco presentadas por Von Keyserlingk et al. (1996). La fracción degradable rápida a, como también la $\mathrm{a}+\mathrm{b}$, fueron mayores en el FS $(\mathrm{P}<0,05)$ que en el FD. Si bien, la fracción soluble a en la PB presentaron significativamente $(\mathrm{P}<0,01)$ mayores valores que en la PA, no hubo diferencia significativa $(\mathrm{P}>0,05)$ entre éstas para degradación máxima y para la degradabilidad efectiva. La interacción para la frac-

CUADRO 1. Características del forraje disponible a ras del suelo (FD) y del forraje aparentemente consumido (FS), obtenidas a la pradera baja (PB) y alta (PA).

\begin{tabular}{lccccc}
\hline Características del forraje & \multicolumn{2}{c}{ FD } & & \multicolumn{2}{c}{ FS } \\
\cline { 2 - 3 } \cline { 5 - 6 } & PB & PA & & PB & PA \\
\hline Altura de pradera (cm) & 6,4 & 10,7 & 6,4 & 10,7 \\
Material muerto (kg/ha) & 336 & 483 & - & - \\
Material vivo (kg/ha) & 1344 & 2203 & - & - \\
Materia seca (MS, \%) & - & - & 202 & 207 \\
Materia orgánica (MO, g/kg de MS) & 913 & 913 & & 904 & 908 \\
Cenizas totales (CT, g/kg de MS) & 87 & 87 & 96 & 92 \\
Proteína total (PT, g/kg de MS) & 169 & 135 & 226 & 231 \\
Fibra detergente neutro (FDN, g/kg de MS) & 465 & 473 & & 448 & 421 \\
Fibra detergente ácido (FDA, g/kg de MS) & 243 & 245 & 232 & 222 \\
Energía metabólica (EM, MJ/kg de MS) & 11,9 & 11,8 & 12,4 & 12,6 \\
\hline
\end{tabular}

CUADRO 2. Degradabilidad ruminal de la materia seca, en muestras de forrajes disponible (FD) y aparentemente consumida (FS), obtenidas a la pradera baja (PB) y alta (PA).

\begin{tabular}{|c|c|c|c|}
\hline \multirow[t]{2}{*}{ Variables } & \multicolumn{3}{|c|}{ Degradabilidad de la materia seca $^{1}$} \\
\hline & $\mathrm{a}$ & $\mathrm{a}+\mathrm{b}$ & $\mathrm{DE}$ \\
\hline Muestras de forraje & \multicolumn{3}{|c|}{ 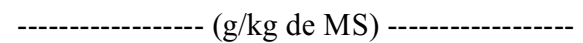 } \\
\hline FD & 299 & 786 & 474 \\
\hline FS & 351 & 850 & 508 \\
\hline Significancia & $* * *$ & $* * *$ & $*$ \\
\hline \multicolumn{4}{|l|}{ Altura de pradera } \\
\hline PA & 327 & 814 & 497 \\
\hline PB & 323 & 822 & 485 \\
\hline Significancia & NS & NS & NS \\
\hline e.e.d. ${ }^{2}$ & 5,4 & 8,1 & 13,0 \\
\hline Interacción & & & \\
\hline Muestras de forraje $\mathrm{x}$ altura de pradera & NS & NS & NS \\
\hline
\end{tabular}

${ }^{1}$ a: fracción soluble (tiempo zero); a+b: degradación máxima potencial; DE: degradabilidade mineral efectiva.

2 Error estándar de la diferencia entre los promedios.

NS $*$ y *** Non-significativo y significativo cuando $\mathrm{P}<0,05$ y $\mathrm{P}<0,001$, respectivamente 
ción soluble fue significativa $(\mathrm{P}<0,01)$ para tipo de forraje y altura de pradera.

Las muestras de FD mostraron concentraciones más bajas en proteína total (Cuadro 1), en la fracción de soluble a y en degradabilidad efectiva, que en las muestras aparentemente consumidas en pastoreo. Los valores más bajos en proteína total del FD pudo deberse a un aumento de la proporción de tallos con

CUADRO 3. Degradabilidad ruminal de la materia orgánica, en muestras de forrajes disponible (FD) y aparentemente consumido (FS), obtenidas a la pradera baja (PB) y alta (PA).

\begin{tabular}{|c|c|c|c|}
\hline \multirow[t]{2}{*}{ Variables } & \multicolumn{3}{|c|}{ Degradabilidad de la materia orgánica $^{1}$} \\
\hline & $\mathrm{a}$ & $\mathrm{a}+\mathrm{b}$ & $\mathrm{DE}$ \\
\hline Muestras de forraje & \multicolumn{3}{|c|}{ - } \\
\hline FD & 304 & 805 & 490 \\
\hline FS & 376 & 855 & 529 \\
\hline Significancia & $* * *$ & $* * *$ & $* *$ \\
\hline \multicolumn{4}{|l|}{ Altura de pradera } \\
\hline PA & 342 & 826 & 515 \\
\hline PB & 337 & 834 & 504 \\
\hline Significancia & NS & NS & NS \\
\hline e.e.d. ${ }^{2}$ & 3,3 & 7,6 & 11,6 \\
\hline Interacción & & & \\
\hline Muestras de forraje $\mathrm{x}$ altura de pradera & NS & NS & NS \\
\hline
\end{tabular}

${ }^{1}$ a: fracción soluble (tiempo zero); a+b: degradación máxima potencial; DE: degradabilidad ruminal efectiva.

2 Error estándar de la diferencia entre los promedios.

NS, ** y *** Non-significativo y significativo cuando $\mathrm{P}<0,01$ y $\mathrm{P}<0,001$, respectivamente

CUADRO 4. Degradabilidad ruminal del nitrógeno, en muestras de forrajes disponible (FD) y aparentemente consumido (FS), obtenidas a la pradera baja (PB) y alta (PA).

\begin{tabular}{|c|c|c|c|}
\hline \multirow[t]{2}{*}{ Variables } & \multicolumn{3}{|c|}{ Degradabilidad del nitrógeno $^{1}$} \\
\hline & $\mathrm{a}$ & $\mathrm{a}+\mathrm{b}$ & $\mathrm{DE}$ \\
\hline Muestras de forraje & \multicolumn{3}{|c|}{ 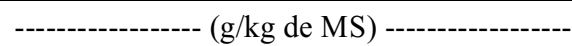 } \\
\hline FD & 250 & 676 & 351 \\
\hline FS & 301 & 850 & 419 \\
\hline Significancia & $* *$ & $* * *$ & $* * *$ \\
\hline \multicolumn{4}{|l|}{ Altura de pradera } \\
\hline PA & 265 & 749 & 365 \\
\hline PB & 285 & 776 & 406 \\
\hline Significancia & ** & NS & NS \\
\hline e.e.d. ${ }^{2}$ & 6,4 & 15,4 & 10,0 \\
\hline Interacción & & & \\
\hline Muestra de forraje $\mathrm{x}$ altura de pradera & $* *$ & NS & NS \\
\hline Interacción para la fracción soluble a & \multicolumn{2}{|c|}{ Altura de pradera } & \\
\hline Muestra de forraje & PA & PB & \\
\hline FD & 253 & 250 & \\
\hline FS & 281 & 321 & \\
\hline e.e.d. ${ }^{2}$ & \multicolumn{2}{|c|}{9,0} & \\
\hline
\end{tabular}


CUADRO 5. Degradabilidad ruminal del fibra detergente neutro (FDN), en muestras de forrajes disponible (FD) y aparentemente consumido (FS), obtenidas la pradera baja (PB) y alta (PA).

\begin{tabular}{|c|c|c|c|}
\hline \multirow[t]{2}{*}{ Variables } & \multicolumn{3}{|c|}{ Degradabilidad del FDN $^{1}$} \\
\hline & A & $\mathrm{a}+\mathrm{b}$ & $\mathrm{DE}$ \\
\hline Muestras de forraje & \multicolumn{3}{|c|}{ 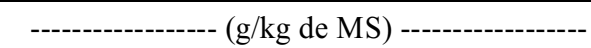 } \\
\hline FD & 457 & 641 & 260 \\
\hline FS & 233 & 698 & 259 \\
\hline Significancia & $* *$ & $* * *$ & NS \\
\hline \multicolumn{4}{|l|}{ Altura de pradera } \\
\hline PA & 343 & 667 & 263 \\
\hline PB & 347 & 672 & 256 \\
\hline Significancia & NS & NS & NS \\
\hline \multirow{2}{*}{\multicolumn{4}{|c|}{$\begin{array}{l}\text { e.e.d. } \\
\text { Interacción }\end{array}$}} \\
\hline & & & \\
\hline Muestras de forraje $\mathrm{x}$ altura de pradera & NS & NS & NS \\
\hline
\end{tabular}

1 a: fracción soluble (tiempo zero); a+b: degradación máxima potencial; DE: degradabilidad ruminal efectiva.

2 Error estándar de la diferencia entre los promedios.

NS, ** y *** Non-significativo y significativo cuando $\mathrm{P}<0,01$ y $\mathrm{P}<0,001$, respectivamente.

un menor porcentaje de proteína total, en relación al nivel proteico de las hojas. Además, Steg et al. (1994) agregan que es posible esperar una mayor fracción a cuando el forraje presenta una mayor proporción de nitrógeno no proteico, situación esperable en el FS. Van Straalen \& Tamminga (1990) establecieron que la degradabilidad de la proteína en el forraje puede ser influida por diferentes parámetros como el nivel de fertilización y la madurez de la pradera.

Por otra parte, la fracción proteica no-degradable del FD, fue mayor $(32,4 \%)$ que en las muestras aparentemente consumidas (15\%) (Cuadro 3). Una explicación para estas diferencias puede ser el efecto de avance en madurez de la pradera, observado como cambios en la proporción del tallo/hoja y la proporción de materia muerta entre ambas muestras de forraje evaluadas. Van Vuuren et al. (1991) y Steg et al. (1994) reportaron que un aumento en la proporción de nitrógeno unido a las paredes celulares puede contribuir a una degradabilidad más baja del nitrógeno en muestras de forraje, y por consiguiente no disponible para la digestión ruminal. Por lo tanto, la mayor degradabilidad de las muestras de pasto aparentemente consumidas fue probablemente el resultado de que la proteína de la hoja es mayormente degradable en comparación con la del FD en su con- junto. También, dicho efecto sería observado en la altura baja del FS, donde aparentemente habría habido una mayor proporción de tejido foliar, con una consiguiente mayor nivel de la fracción a, que en la PA del forraje aparentemente consumido.

Las características de degradación de FDN de los forrajes se entrega en el Cuadro 5. Van Vuuren et al. (1991) reportan un aumento en la fracción indigestible del FDN contenida en los forrajes, asociado a un aumento de su lignificación. En el presente trabajo no se midió el porcentaje de lignina en los forrajes, sin embargo, el FD presentó mayores valores de FDN y FDA, los que podrían a su vez contener un mayor grado de lignificación que en el forraje aparentemente consumido. Sin embargo, Steg et al. (1994) indicaron en su trabajo que la degradabilidad de la fracción de FDN no fue afectada por la estación del año, a pesar de que hubo un claro aumento estacional en el contenido de la pared celular de la pradera.

\section{CONCLUSIONES}

1. El forraje aparentemente consumido por los bovinos difiere en su cinética degradativa ruminal al forraje disponible a ras de suelo. 
2. La altura de muestreo no presenta un efecto significativo ni consistente sobre la dinámica degradativa.

\section{REFERENCIAS}

AGRICULTURAL AND FOOD RESEARCH COUNCIL. Energy and protein requirements of ruminants. An advisory manual prepared by the AFRC Technical Committee on Responses to Nutrients. Wallingford : CAB International, 1993. $159 \mathrm{p}$.

ASSOCIATION OF OFFICIAL ANALYTICAL CHEMISTS. Official Methods of Analysis of the Association of Official Analytical Chemists. 13.ed. Washington, 1980. 978p.

BALDE, A.T.; VANDERSALL, J.H.; ERDMAN, A.R.; REEVES III, J.B.; GLENN, P.B. Effect of stage of maturity of alfalfa and orchard grass on in situ dry matter and crude protein degradability and amino acid composition. Animal Feed Science and Technology, Amsterdam, v.44, p.29-43, 1993.

BLACK, J.L. Nutrition of the grazing ruminant. New Zealand Society of Animal Production. Proceedings, Hamilton, v.50, p.7-34, 1990

DUDZINKI, M.L.; ARNOLD, G.W. Comparisons of diet of sheep and cattle grazing together on sown pasture on the southern tablelands of New South Wales by principle components analysis. Australian Journal of Agricultural Research, Melbourne, v.24, p.899912, 1973

FORBES, J.M. The voluntary food intake of farm animals. London : Butterworth, 1986. 206p.

GENSTAT 5 COMMITTEE. GenStat reference manual. Oxford : Clarendon, 1987. 300p.

GIVENS, D.I.; EVERINGTON, J.M.; ADAMSON, A.H The nutritive value of Spring-grown herbage produced on farms throughout England \& Wales over 4 years. III. The prediction of energy values from various laboratory measurements. Animal Feed Science and Technology, Amsterdam, v.27, p.185196, 1990 .

GOERING, H.K.; VAN SOEST, P.J. Forage fiber analysis. Washington, DC : Agriculture Research Service, USDA, 1970. 19p. (Agriculture Handbook, No. 379).

INGLATERRA. Ministry of Agriculture Fisheries and Food. Prediction of energy value of compound feedingstuffs for farm animal. Alnwick, 1993. 6p. (MAFF Publications. Booklet, 1285).

JEWELL, S.N.; OSBORNE, E.D.; CAMPLING, R.C. Effect of species and cultivar of cereal straws on response to ammoniation as measured by a cellulase digestibility technique. Journal of Natural Institute Agriculture \& Botany, v.17, p.199-208, 1986.

McDONALD, I.M. A revised model for the estimation of protein degradability in rumen. Journal of Agricultural Science, Cambridge, v.96, p.251-252, 1981

ORSKOV, E.R.; McDONALD, I.M. Estimation of protein degradability in the rumen from incubation measurement weighted according to rate of passage. Journal of Agricultural Science, Cambridge, v.96, p.499-503, 1979.

PAINE, C.A.; CRANSHAW, R.; BAKER, W.P. A complete exchange method for in sacco estimation of rumen degradability on a routine basis. In: SYMPOSIUM ON FORAGE PROTEIN IN RUMINANT ANIMAL PRODUCTION, 1981, Bodington Hall. Proceedings. Thames Ditton British Society of Animal Production, 1982. p.177178. (BSAP. Occasional Publication, 6).

PULIDO, R.G. Interactions of pasture conditions, concentrate supplementation and milk yield level in relation to dairy cow performance and behaviour. Wye College : University of London, 1997. 264p. Ph.D. Thesis.

STEG, A.; VAN STRAALEN, W.M.; HINDLE, V.A.; WENSIK, W.A.; DOOPER, F.M.H.; SCHILS, R.L.M. Rumen degradation and intestinal digestion of grass and clover at two maturity levels during the season in dairy cows. Grass and Forage Science, Oxford, v.49, p.378-390, 1994.

VAN STRAALEN, W.M.; TAMMINGA, S. Protein degradation of ruminant diets. In.: WISEMAN, J.; COLE, D.J.A. (Eds.). Feedstuff evaluation. London : Butterworths, 1990. p.55-72.

VAN VUUREN, A.M.; TAMMINGA, S.; KETELAAR, R.S. In sacco degradation of organic matter and crude protein of fresh grass (Lolium perenne) in the rumen of grazing dairy cows. Journal of Agricultural Science, Cambridge, v.116, p.429-436, 1991.

VON KEYSERLINGK, M.A.G.; SWIFT, M.L.; PUCHALA, R.; SHELFORD, J.A. Degradability characteristics of dry matter and crude protein of forages in ruminants. Animal Feed Science and Technology, Amsterdam, v.57, p.291-311, 1996.

Pesq. agropec. bras., Brasília, v.35, n.5, p.1003-1009, maio 2000 